\title{
VIABILIDADE DE UM PROJETO FLORESTAL DE Eucalyptus grandis CONSIDERANDO O SEQÜESTRO DE CARBONO
}

\author{
Romualdo Maestri ${ }^{1}$ \\ Carlos Roberto Sanquetta ${ }^{2}$ \\ Sebastião do Amaral Machado ${ }^{3}$ \\ José Roberto Soares Scolforo ${ }^{4}$ \\ Ana Paula Dalla Côrte ${ }^{5}$
}

\begin{abstract}
RESUMO
Foram avaliados cenários econômicos para créditos do seqüestro de carbono em florestas de Eucalyptus grandis na região sudeste e nordeste do Brasil. Curvas de produtividade volumétrica e de carbono em diferentes frações foram obtidas. A análise identificou taxas internas de retorno (TIR) de no máximo 7,7\% ao ano para sítios de alta produtividade $\left(38,2 \mathrm{~m}^{3} \cdot \mathrm{ha}^{-1} \cdot \mathrm{ano}^{-1}\right)$. Se a biomassa total da floresta (radicular e aérea) for considerada, a TIR acima de $6 \%$ ao ano são possíveis mesmo para produtividades menores $\left(30 \mathrm{~m}^{3} \cdot \mathrm{ha}^{-1}\right.$.ano $\left.{ }^{-1}\right)$. Assim, seria necessária uma remuneração de US\$ $25,00 /$ t para que o empreendimento atingisse remuneração de $6 \%$ ao ano quando o seqüestro de carbono fosse objetivo de negócio e para produtividade volumétrica de $24 \mathrm{~m}^{3}$. $\mathrm{ha}^{-1}$.ano $\mathrm{au}^{-1}$ ou US\$ $15,50 / \mathrm{t}$ para produtividade de $38,2 \mathrm{~m}^{3} \cdot \mathrm{ha}^{-1} \cdot \mathrm{ano}^{-1}$. Se considerada a venda da porção lenhosa do tronco, então seria possível auferir a mesma rentabilidade ao preço de US\$12,00/t para produtividades acima de $34,7 \mathrm{~m}^{3} . \mathrm{ha}^{-}$ ${ }^{1}$.ano ${ }^{-1}$.

Palavras-chave: biomassa florestal, economia, mudanças climáticas, produtividade, taxa interna de retorno.
\end{abstract}

\section{FEASIBILITY OF A FOREST PROJECT OF Eucalyptus grandis TAKING INTO ACCOUNT CARBON} SINK

\begin{abstract}
The purpose of this study was to investigate some economic scenarios for carbon sink through Eucalyptus grandis stands forest projects in southeastern and northeastern Brazil. Below and above ground stored carbon and biomass were obtained through dbh class growth and yield empirical models as functions of different environmental conditions. The analisys was identificed internal rate of return (IRR) above $7.7 \%$ per annum for stands at high productivity sites $\left(38.2 \mathrm{~m}^{3} \cdot \mathrm{ha}^{-1} \cdot \mathrm{year}^{-1}\right)$. If below and aboveground stored carbon is considered, an IRR greater than $6 \%$ pa. may be achieved even at lower productivity sites $\left(30 \mathrm{~m}^{3} \cdot \mathrm{ha}^{-1}\right.$.year $\left.{ }^{-1}\right)$. It would be necessary a carbon price of US\$ $25.00 / \mathrm{t}$ to match an IRR of $6 \%$ pa at low productivity sites $\left(24 \mathrm{~m}^{3}\right.$.ha $\mathrm{a}^{-1}$.year $\left.{ }^{-1}\right)$ and US\$ $15.50 / \mathrm{t}$ at high ones $\left(38.2 \mathrm{~m}^{3} \cdot \mathrm{ha}^{-1}\right.$.year $\left.{ }^{-1}\right)$. Additionally, if stem biomass was also traded in the wood market, carbon price of US\$12.00/t would allow to achieve the same IRR.
\end{abstract}

Keywords: forest biomass, economics, climatic changes, productivity, internal rate of return

\section{INTRODUÇÃO}

Há uma crescente evidência de que a Terra está passando por um processo de aquecimento, que poderia produzir efeitos climáticos e afetar a vida humana no futuro. Em 1992 foi realizada a Convenção das Nações Unidas sobre Mudanças Climáticas, propondo ações, principalmente nos países

industrializados, para estabilizar a concentração atmosférica dos gases geradores do efeito estufa (GEE). Segundo Rocha (2002) a Convenção do Clima entrou em vigor em 21 de março de 1994 e conta atualmente com 186 países. Desde então, as partes reuniram-se regularmente (Kyoto-97, Buenos

\footnotetext{
${ }^{1}$ Eng. Florestal,M.Sc., Dr. em Ciências Florestais UFPR, Pesquisador Sênior da Aracruz Florestal S.A. rmaestri@aracruz.com.br

${ }^{2}$ Eng. Florestal, M.Sc., Ph.D. Professor do Departamento de Ciências Florestais da UFPR. sanqueta@floresta.ufpr.br

${ }^{3}$ Eng. Florestal, M.Sc.,Dr. Professor do Departamento de Ciências Florestais da UFPR. sammac@floresta.ufpr.br

${ }^{4}$ Eng. Florestal, M.Sc., Dr. Professor do Departamento de Engenharia Florestal da UFLA. scolforo@ufla.br

${ }^{5}$ Eng. Florestal, Mestranda em Ciências Florestais da UFPR, Prof ${ }^{4}$. do Depto de Ciências Florestais da UFPR. anacorte@floresta.ufpr.br

Recebido para publicação: 31/08/2004

Aceito para publicação: 07/12/2004
} 
Aires-98, Bonn-99, Haia-2000 e Bonn-2001), para discutir o assunto e encontrar soluções para o problema. Seguramente o encontro de Kyoto foi o marco das discussões, onde as nações signatárias concordaram com a meta de redução global de 5,2\% na emissão de gases havida em 1990, num prazo de até 2012

Um caminho vislumbrado também no encontro de Kyoto foi a possibilidade de comercialização dos créditos de carbono entre países através do incremento do seqüestro de carbono em reservas terrestres, sobretudo através de projetos de florestamento e reflorestamento. Tal possibilidade abriu novas perspectivas para o setor florestal, que poderá se beneficiar dessa decisão. Segundo Gannel (1999), o argumento básico em favor do plantio de árvores para seqüestro de carbono é que permitiria um lastro de tempo necessário para encontrar soluções de longo prazo para atender o suprimento de energia mundial sem pôr em perigo o sistema climático atenuando o efeito da concentração de $\mathrm{CO}_{2}$ de forma relativamente barata.

O mercado de carbono ainda não está regulamentado, dado que o Protocolo de Kyoto não está ratificado plenamente, ficando a comercialização do carbono ainda sem regras definidas. Contudo, as negociações para formação deste mercado estão em franco desenvolvimento. De acordo com Rocha (2002), várias empresas já estão em busca de "early credits" (créditos antecipados) e em vários países estão sendo criados mercados para comercialização dos certificados de redução de emissões (CRE). O valor do carbono seqüestrado está estimado entre US\$ 1,00 e 50,00 por tonelada, com tendência maior para valores entre US\$ 7,00 e 14,00. Goldenberg (1999), citado por Rocha (2002), estima que os recursos a serem negociados apresentam um potencial atual próximo a US\$ 3 bilhões por ano, elevando-se para a faixa entre US\$ 5 e 17 bilhões anuais a partir de 2010, quando os mecanismos para este comércio estiverem devidamente definidos e aceitos pela comunidade internacional.

Os projetos de florestamento e reflorestamento em países não participantes do acordo de Kyoto, como é o caso do Brasil, estão aguardando a definição de regras mais claras que permitam vislumbrar investimentos externos pela troca por certificados de redução de emissões (CRE). Algumas bases para elegibilidade de projetos de seqüestro de carbono estão sendo discutidas nos encontros entre os países membros, destacando-se a premissa da adicionalidade, que prevê que um projeto deve elevar a taxa de seqüestro além dos níveis atuais, ou seja, um projeto de carbono deve contemplar um seqüestro adicional (Ribeiro et.al, 2002).

Do ponto de vista prático, a determinação da quantidade de carbono a ser seqüestrada depende da variável biomassa, a qual, segundo Sanquetta (2002) precisa ser estimada de forma fidedigna, caso contrário não haverá consistência na quantificação do carbono fixado nos ecossistemas florestais. Inúmeros estudos do conteúdo de carbono em florestas vêm sendo desenvolvidos recentemente e a maioria versa sobre amostragem destrutiva da biomassa e ajuste de modelos alométricos para sua estimativa. A quantificação das frações raiz, tronco e folha se reveste de grande importância e, metodologias de amostragem e modelagem para sua inferência, tornam-se prioritárias para embasar as tomadas de decisão.

Gutiérrez \& Lopera (2001) afirmam que para realizar projetos florestais de captura de $\mathrm{CO}_{2}$ é necessário desenvolver metodologias que permitam medir e quantificar de maneira confiável e a baixo custo o carbono armazenado, mas também predizer seu comportamento em qualquer momento durante o crescimento da plantação. Assim, quando a floresta ainda não é existente, projetar a curva de crescimento esperada, torna-se a base para negociação de créditos de carbono entre as partes interessadas. Em termos de planejamento na definição dos locais mais aptos para efetivação de projetos de seqüestro de carbono, sistemas de predição de produção que levem em consideração as condições ambientais locais como limitantes ao crescimento, se constituem em ferramentas igualmente úteis.

O presente trabalho tem por objetivo apresentar cenários de viabilidade econômica de um projeto florestal de povoamentos clonais de Eucalytpus grandis levando em conta o eventual benefício decorrente do serviço ambiental do seqüestro de carbono. Como tais 
povoamentos apresentam grande influência do ambiente, sobretudo do regime hídrico, é analisada a influência que tal fator determina na produtividade florestal, no seqüestro de carbono e na rentabilidade do projeto.

\section{MATERIAL E MÉTODOS}

Localização

A área de estudo, de propriedade da empresa Aracruz Celulose S.A. está localizada na parte leste e nordeste do Estado do Espírito Santo e no extremo sul da Bahia, conforme apresenta a figura 1 .

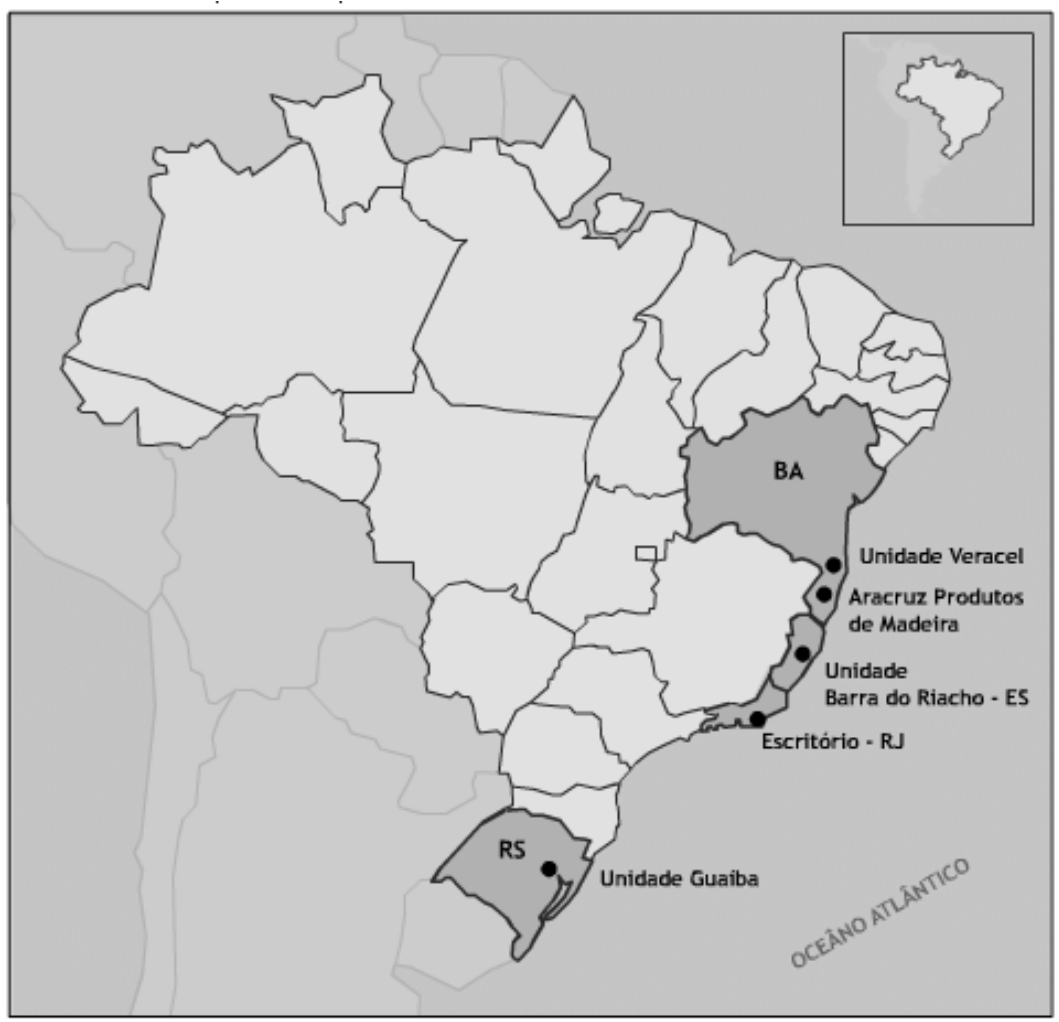

Figura 1: Localização das áreas de estudo

Figure 1: Localization of studied area

\section{Condições ambientais da região de estudo}

A região de estudo está compreendida entre as longitudes $39^{\circ} 05^{\prime} \mathrm{W}$ e $40^{\circ} 20^{\prime} \mathrm{W}$ e entre as latitudes $17^{\circ} 15^{\prime} \mathrm{S}$ e $20^{\circ} 15^{\prime} \mathrm{S}$, caracterizada, segundo a classificação climatológica de Köppen, como de clima tropical úmido a quente, com temperaturas de inverno acima dos $18^{\circ} \mathrm{C}$.

Predominam nas áreas do Espírito Santo os grupos de solos: Latossolo VermelhoAmarelo distrófico, coeso, relevo plano e suave ondulado (platôs litorâneos) e o Podzólico Vermelho-Amarelo abrupto, relevo plano e suave ondulado (platôs litorâneos), além de Podzol.
Para a região do extremo sul da Bahia, os principais grupos de solo, são: Latossolo com baixo teor de ferro, relacionado com sedimentos do tipo Tabuleiro, cor amarela, e os Podzóis relacionados a sedimentos marinhos, constituídos por arenitos não consolidados, de fertilidade muito baixa e má condição de drenagem e com textura arenosa.

Referências e estrutura geral da modelagem

Neste trabalho foram empregadas metodologias desenvolvidas com base em dados colhidos na própria região de estudo por vários autores. Maestri (2003) desenvolveu modelos de crescimento e produção volumétricos baseados em projeções das características dos povoamentos e de sua 
estrutura diamétrica para diferentes condições climáticas. Thiersch (2002) desenvolveu modelos para projeção da densidade básica da madeira a partir de variáveis do povoamento e da árvore. $O$ fato de a base genética ser de material clonal demonstrou grande estabilidade na explicação da variável densidade da madeira como função da idade, sítio, diâmetro e altura da árvore. Neves (2002) estudou a partição de biomassa entre os vários compartimentos da floresta e seus respectivos teores de carbono para as mesmas condições deste estudo. Em conjunto, o resultados destes três trabalhos permitiram a estruturação de um

Modelo para projeção volumétrica

Para a projeção da produção do volume de madeira do tronco das árvores em

Hdom $=45,5823\left(\right.$ Modi $\left._{A}\right)\left[1-\exp (-0,0901 \text { Id) }]^{0,6955}\right.$

$\left(\mathrm{R}^{2}=78,3 \%\right.$; $\left.\mathrm{Sxy}=10,5 \%\right)$

sendo:

$\operatorname{Modi}_{A}=0,2002 \operatorname{Ln}(P P)+0,0348 \operatorname{Ln}(E T P d p m)$

$N=N i(1-S / 100) \exp [-(I d-1) / 0,15896]^{0,001204}$

$\left(\mathrm{R}^{2}=69,1 \%\right.$ Sxy $\left.=6,0 \%\right)$

$\operatorname{Ln}(A B)=-4,994-0,83836(1 / I d)+1,09439 \operatorname{Ln}(N)+0,66652 \operatorname{Ln}($ Hdom $)$

$\left(\mathrm{R}^{2}=99,9 \%\right.$ Sxy $\left.=7,9 \%\right)$

onde:

Hdom = altura dominante (m);

$\operatorname{Modi}_{A}=$ coeficiente modificador do parâmetro assintótico do modelo de Hdom, em função das variáveis ambientais;

$I d=$ idade do povoamento (anos);

$P P=$ precipitação média mensal histórica $(\mathrm{mm})$;

$E T P d p m=$ desvio padrão da média mensal da evapotranspiração potencial $(\mathrm{mm})$;

$N=$ número corrente de árvores por hectare;

$\mathrm{Ni}=$ número de árvores plantadas por hectare;

$S=$ porcentagem de mortalidade no primeiro ano;

$A B=$ área basal $\left(\mathrm{m}^{2} / \mathrm{ha}\right)$.

Para estimativa da variância dos diâmetros e os diâmetros mínimo e máximo do povoamento, valeu-se dos modelos

desenvolvidos por Thiersch (2002), que trabalhou com o mesmo material genético na mesma área de estudo, a saber:

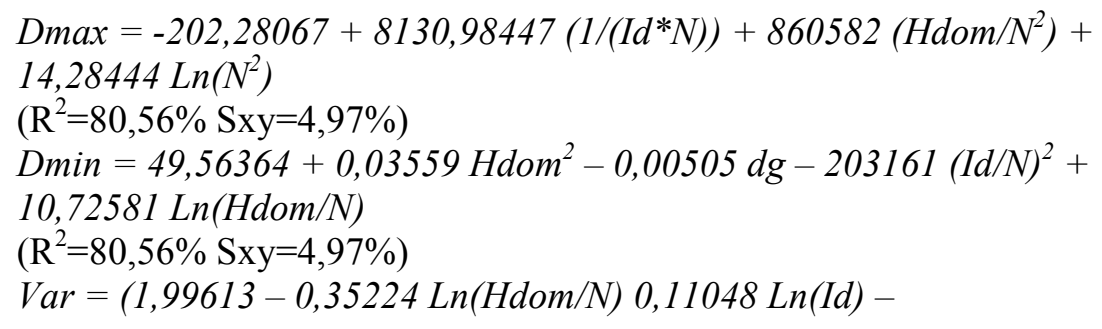


$0,59124 \operatorname{Ln}(A B N)-0,01553 \operatorname{Dmin}^{2}+0,31774$ Dmax $)^{2}$

$\left(\mathrm{R}^{2}=69,91 \%\right.$ Sxy $\left.=9,08 \%\right)$

onde:

Dmax = diâmetro máximo do povoamento $(\mathrm{cm})$;

Dmin = diâmetro mínimo do povoamento $(\mathrm{cm})$;

$D g=$ diâmetro quadrático $(\mathrm{cm})=[A B 40000 /(N \pi)] 0,5$;

Var $=$ variância dos diâmetros do povoamento $\left(\mathrm{cm}^{2}\right)$.

Com o conjunto de modelos apresentados acima é possível, então, fazer a projeção no tempo, das características do povoamento florestal, levando-se em conta a precipitação histórica do local. Isto significa dizer que diferentes condições climáticas interferem na expectativa de produção e, conseqüentemente, na quantidade de carbono a ser seqüestrado pelo povoamento.

A estimativa volumétrica é baseada na distribuição de diâmetros obtida através da recuperação dos coeficientes da função Weibull pelo método de momentos. A forma da função densidade de probabilidade de Weibull é dada por:

$$
f(x)=\left(\frac{c}{b}\right)\left(\frac{D A P-a}{b}\right)^{c-1} e^{-\left(\frac{D A P-a}{b}\right)^{c}}
$$

Os parâmetros " $a$ ", " $b$ " e " $c$ " definem a locação, escala e forma da distribuição. $O$ método de momentos recupera o parâmetro de forma " $c$ " da função probabilística por processo iterativo, relacionado-o ao coeficiente de variação em diâmetro do povoamento, segundo metodologia encontrada em Scolforo (1998) e Maestri (2003).

$$
C V_{D A P}=\frac{\sigma}{D A P m e d}=\frac{\sqrt{\left(\Gamma\left(1+\frac{2}{c}\right)-\Gamma^{2}\left(1+\frac{1}{c}\right)\right.}}{\Gamma\left(1+\frac{1}{c}\right)}
$$

onde:

$C V_{D A P}=$ coeficiente de variação do DAP;

$\sigma=$ desvio padrão do diâmetro ou raiz quadrada da variância do diâmetro $(\mathrm{cm})$;

DAPmed $=$ diâmetro médio aritmético do povoamento $(\mathrm{cm})$;

$\Gamma=$ função gama;

$c=$ parâmetro forma da função probabilística de Weibull.

Uma vez recuperado o parâmetro da

forma, então o parâmetro de escala " $b$ " é

obtido através da relação:

$$
b=\frac{\text { DAPmed }}{\Gamma\left(1+\frac{1}{c}\right)}
$$

onde:

$b=$ parâmetro escala da função probabilística de Weibull;

DAPmed = diâmetro médio aritmético do povoamento $(\mathrm{cm})$.

O parâmetro de locação " $a$ " da função Weibull é definido também por processo iterativo ajustando a posição da distribuição de diâmetros, de forma a compatibilizar a área basal resultante com aquela estimada pelo modelo de área basal apresentado acima.
Uma vez que a metodologia para estimativa das características do povoamento (altura dominante, sobrevivência de árvores, área basal, diâmetros mínimo e máximo e a variância do DAP) e de sua respectiva distribuição diamétrica estejam definidas, o 
passo seguinte consiste no uso de duas funções adicionais aplicadas a cada classe diamétrica: a) modelo para estimativa da altura total das árvores; b) modelo para estimativa do volume de madeira do tronco, através das equações hipsométrica e de afilamento:

$$
H t_{i}=e^{\beta_{1}+\beta_{2} \operatorname{Ln}(H d o m)+\beta_{3} \operatorname{Ln}\left(\frac{D g}{D A P m e d}\right)+\beta_{4}\left(\frac{1}{I d . D A P_{i}}\right)+\beta_{5}\left(\frac{1}{D A P_{i}}\right)}
$$

onde:

$H t_{i}=$ altura total da classe de $D A P_{i}(\mathrm{~m})$;

$D A P_{i}=$ centro da classe de diâmetro $(\mathrm{cm})$;

$H d=$ altura das árvores dominantes do povoamento (m);

$D g$ = diâmetro quadrático do povoamento $(\mathrm{cm})$;

DAPmed = diâmetro médio aritmético do povoamento $(\mathrm{cm})$;

$\beta_{1}=0,43865$;

$\beta_{2}=0,890308$

$\beta_{3}=-0,30468$

$\beta_{4}=0,735183$

$\beta_{5}=-2,521378$

$\mathrm{R}^{2}=97,4 \%$ e Sxy $=1,90 \%$.

$$
\frac{d_{i}}{D A P_{j}}=\beta_{0}+\beta_{1}\left(\frac{h_{i}}{H t_{j}}\right)^{p 1}+\beta_{2}\left(\frac{h_{i}}{H t_{j}}\right)^{p 2}+\ldots+\beta_{n}\left(\frac{h_{i}}{H t_{j}}\right)^{p n}+e_{i}
$$

onde:

$d_{i}=$ diâmetro sem casca do fuste numa altura correspondente $h_{i}$;

$h_{i}=$ altura do fuste correspondente ao diâmetro $d_{i}$;

$H t_{j}=$ altura total da árvore da classe de diâmetro j;

$D A P_{i}=$ diâmetro com casca à altura do peito da classe de diâmetro j;

$\beta_{o}=1,14308$;

$\beta_{1}=0,53878 ; p 1=0,00001 ;$

$\beta_{2}=1,96379 ; p 2=0,6$;

$\beta_{3}=-1,80996 ; p 3=0,2$;

$\beta_{4}=-1,54403 ; p 4=1,0$;

$\beta_{5}=-0,29115 ; p 5=5,0$;

$\beta_{6}=0,008382 ; p 5=60,0$;

$\mathrm{R}^{2}=98,6 \%$ e Sxy $=6,76 \%$.

Os volumes totais e comerciais de madeira sem casca são obtidos pela integração da função polinomial (12), considerando o tronco como um sólido de revolução e as

$$
\begin{aligned}
& V T s c=\frac{\pi}{4} \int_{0}^{H t} d i^{2} d x \\
& V C s c=\frac{\pi}{4} \int_{0}^{h i} d i^{2} d x
\end{aligned}
$$

Modelo para projeção da densidade da madeira

A densidade básica de árvores individuais foi relacionada às características do estimativas são obtidas de uma posição inferior (no caso 0 , que corresponde à base da árvore) até qualquer altura superior $\left(h_{i}\right)$ do tronco (equações 13 e 14).

povoamento idade e altura dominante $\mathrm{e}$ também, ao DAP e à altura total da árvore, gerando a seguinte equação (Thiersch, 2002):

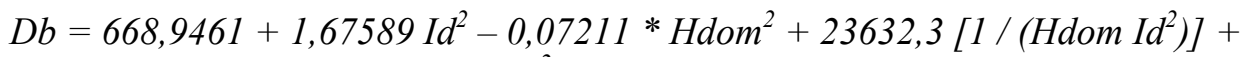
1,64457 DAP - 87,93211 (DAP/Id $\left.{ }^{2}\right)-4155,096(1 / H t)$ 
onde:

$D b=$ densidade básica da madeira $\mathrm{em} \mathrm{kg} / \mathrm{m}^{3}$;

$\mathrm{R}^{2}=80,26 \%$ e Sxy $=2,89 \%$.

A equação (15) foi ajustada com base em 108 árvores coletadas em povoamentos clonais de eucalipto na região de estudo, nas idades de 3, 4, 5 e 6 anos e para índices de sítio 23, 26 e $29 \mathrm{~m}$ (idade índice $=5$ anos). Em cada situação 3 árvores foram abatidas cobrindo a distribuição de DAP do povoamento e uma amostra de cavacos de madeira do tronco, possibilitou a determinação da densidade básica em laboratório.

Alocação de biomassa e teor de carbono

Para estimar a alocação de biomassa e o teor de carbono foram empregadas as informações médias por fração reportadas em Neves (2000) e análises do teor de carbono via pirólise, conforme apresentado na tabela 1.

Tabela 1: Alocação relativa da biomassa em povoamentos clonais de Eucalyptus grandis Table 1: Relative biomass placement in Eucalyptus grandis clonal stands

\begin{tabular}{lcc}
\hline Compartimento & Peso relativo total $(\%)$ & Teor de Carbono $(\mathrm{g} / \mathrm{kg})$ \\
\hline Copa (folhas e galhos) & 3,4 & 525,0 \\
Lenho & 73,7 & 506,6 \\
Casca & 6,8 & 479,6 \\
Raiz & 10,8 & 507,0 \\
Serapilheira & 5,3 & 477,8 \\
\hline Total & 100,0 & - \\
\hline
\end{tabular}

Fonte: Neves (2000)

Aspecto econômico de um projeto florestal com seqüestro de carbono

Os valores do carbono seqüestrado ainda não encontraram estabilidade de mercado uma vez que as regras para comercialização ainda não estão totalmente definidas. Na literatura encontram-se valores em ampla faixa, mas muitos autores, segundo Rocha (2002), apontam que o valor médio está ao redor de US\$ 12,00 por tonelada de carbono reduzido.

Procedeu-se, então, com uma avaliação da atratividade econômica de um empreendimento florestal constituído para seqüestrar carbono para diferentes condições de crescimento determinadas pela situação climática histórica de cada local. Através das equações apresentadas anteriormente, foram definidas quatro classes de produtividade em volume (incremento médio anual aos 7 anos -
IMA7) em função da precipitação anual: a) $800 \mathrm{~mm}$ - IMA7 $\left.=24,0 \mathrm{~m}^{3} \mathrm{sc}^{3} \cdot \mathrm{ha}^{-1} \cdot \mathrm{ano}^{-1} ; \mathrm{b}\right)$ $1.300 \mathrm{~mm}-$ IMA7 $\left.=30,0 \mathrm{~m}^{3} \mathrm{sc}^{\mathrm{s}} \cdot \mathrm{ha}^{-1} \cdot \mathrm{ano}^{-1} ; \mathrm{c}\right)$ $1.800 \mathrm{~mm}$ - IMA7 $\left.=34,7 \mathrm{~m}^{3} \mathrm{sc}^{-h^{-1}} \cdot \mathrm{ano}^{-1} ; \mathrm{d}\right)$ $2.300 \mathrm{~mm}$ - IMA7 $=38,2 \mathrm{~m}^{3} \mathrm{sc} \cdot \mathrm{ha}^{-1} \cdot \mathrm{ano}^{-1}$. Os compartimentos da floresta foram analisados de duas formas quanto à sua atratividade para o seqüestro de carbono: a) somente a porção do lenho até $7 \mathrm{~cm}$ com casca - 6,4 cm sem casca (lenho comercial); b) todos os compartimentos incluindo serapilheira.

Para análise do fluxo de caixa foram adotados custos médios para a eucaliptocultura praticados no Espírito Santo apresentados na tabela 2.

A atratividade do plantio florestal como forma de seqüestro de carbono foi analisada pela taxa interna de retorno (TIR) considerando os níveis de preço: US\$ 7,0/tC, US\$ $12,00 / \mathrm{tC}$ e US $\$ 17,00 / \mathrm{tC}$. 
Tabela 2: Custos silviculturais médios em povoamentos de Eucalyptus

Table 2: $\quad$ Silvicultural average costs in Eucalyptus stands

\begin{tabular}{|c|c|c|}
\hline Ano de Incidência & Operação & Custo (US\$/ha) \\
\hline 0 & Preparo do solo & 150,00 \\
\hline 0 & Plantio & 285,00 \\
\hline 0 & Controle plantas daninhas & 95,00 \\
\hline $1^{\circ}$ Corte Raso & Proteção florestal & 7,50 \\
\hline $1^{\circ}$ Corte Raso & Administração & 35,00 \\
\hline
\end{tabular}

Fonte: Aracruz Florestal S/A (2002) - comunicação pessoal

\section{RESULTADOS E DISCUSSÃO}

\section{Produção florestal}

O clima exerce importante influência sobre a produção florestal de eucaliptais. $\mathrm{Na}$ Tabela 3 são apresentadas as projeções das características dos povoamentos clonais de eucalipto utilizando-se as equações 1 a 15 . As estimativas para locais com maior precipitação pluviométrica anual tenderam a maior capacidade de produtiva comparados aos locais mais secos, concordando com as constatações de Stape (2002), que afirma serem os plantios de eucalipto muito influenciados pelo regime hídrico.

Foram simuladas quatro condições climáticas em função da quantidade anual histórica de chuva da região, ou seja, 800 , $1.300,1.800$ e $2.300 \mathrm{~mm}$, traduzindo em situações variando de relativamente seca até bem suprida de água. A variação da precipitação pluviométrica anual foi determinante na produção em madeira. Aos 7 anos de idade o volume total sem casca variou entre $178 \mathrm{~m}^{3}$ e $277,4 \mathrm{~m}^{3}$ considerando precipitações pluviométricas anuais de $800 \mathrm{~m}$ até $2.300 \mathrm{~mm}$, representando uma diferença de $64 \%$ e uma variação no incremento médio anual de $24 \mathrm{~m}^{3}$ sc.hat ${ }^{-1}$.ano ${ }^{-1}$ até $38,2 \mathrm{~m}^{3}$ sc.ha ${ }^{-}$ ${ }^{1}$.ano ${ }^{-1}$. Certamente essas influências afetam sobremaneira a quantidade de carbono seqüestrado pelos povoamentos.

A densidade básica da madeira apresentou uma tendência crescente em relação à idade, porém sem tendências marcantes em função da capacidade produtiva, variando entre 0,45 e $0,58 \mathrm{~g} / \mathrm{cm}^{3}$ dos 2 aos 10 anos.

\section{Seqüestro de carbono}

Considerando os teores de carbono para cada compartimento da árvore $\mathrm{e}$ serapilheira e, dadas as produções projetadas em função das condições climáticas da Tabela 3 , obtiveram-se curvas de crescimento do carbono a ser seqüestrado nos plantios de eucalipto. A Tabela 4 apresenta a expectativa de peso de carbono e de $\mathrm{CO}_{2}$ por hectare para a idade de 7 anos nas quatro condições de precipitação pluviométrica anual.

Conforme já previsto na Tabela 3, maior quantidade de carbono é esperada ocorrer no tronco das árvores dos povoamentos de eucalipto independente da condição climática existente. A contribuição de copa, casca, raiz e serapilheira é consideravelmente inferior e, no conjunto, representa $26 \%$ do total. 
Viabilidade de um projeto...

Tabela 3: Projeções das características dos povoamentos clonais de Eucalyptus grandis em função do perfil de precipitação pluviométrica anual

Table 3: Prediction of stands characteristics of clonal Eucalyptus grandis stands as a function of annual precipitation

\begin{tabular}{|c|c|c|c|c|c|c|c|c|c|}
\hline Precip. $(\mathrm{mm})$ & Idade & DAP (cm) & Altura (m) & Hdom (m) & AB $\left(\mathrm{m}^{2} / \mathrm{ha}\right)$ & Arv/ha & $\begin{array}{c}\text { Vol } \\
\mathrm{m}^{3} \mathrm{cc} / \mathrm{ha}\end{array}$ & $\begin{array}{c}\text { Vol } \\
\mathbf{m}^{3} \mathbf{s c} / \mathbf{h a}\end{array}$ & $\mathrm{Db}(\mathrm{kg} / \mathrm{t})$ \\
\hline \multirow[t]{9}{*}{800} & 2 & 8,9 & 10,7 & 11,7 & 6,9 & 1075 & 17,3 & 29,3 & 454 \\
\hline & 3 & 11,0 & 13,9 & 15,0 & 10,3 & 1067 & 46,0 & 57,7 & 454 \\
\hline & 4 & 12,5 & 16,5 & 17,8 & 13,3 & 1059 & 76,2 & 88,0 & 457 \\
\hline & 5 & 13,7 & 18,7 & 20,1 & 15,8 & 1051 & 109,4 & 119,5 & 482 \\
\hline & 6 & 14,7 & 20,6 & 22,2 & 18,0 & 1043 & 138,8 & 149,1 & 511 \\
\hline & 7 & 15,5 & 22,3 & 24,0 & 19,9 & 1035 & 168,3 & 178,0 & 542 \\
\hline & 8 & 16,2 & 23,8 & 25,6 & 21,6 & 1027 & 195,9 & 205,8 & 574 \\
\hline & 9 & 16,8 & 25,1 & 27,1 & 23,1 & 1020 & 221,9 & 232,3 & 577 \\
\hline & 10 & 17,3 & 26,3 & 28,4 & 24,4 & 1012 & 248,0 & 257,6 & 579 \\
\hline \multirow[t]{9}{*}{1300} & 2 & 9,4 & 11,9 & 12,9 & 7,7 & 1075 & 21,8 & 34,1 & 457 \\
\hline & 3 & 11,6 & 15,4 & 16,6 & 11,6 & 1067 & 60,5 & 71,8 & 457 \\
\hline & 4 & 13,2 & 18,3 & 19,7 & 14,9 & 1059 & 97,6 & 109,2 & 464 \\
\hline & 5 & 14,5 & 20,8 & 22,3 & 17,7 & 1051 & 138,0 & 148,1 & 490 \\
\hline & 6 & 15,5 & 22,9 & 24,6 & 20,2 & 1043 & 174,2 & 184,4 & 519 \\
\hline & 7 & 16,4 & 24,7 & 26,7 & 22,3 & 1035 & 210,0 & 219,8 & 548 \\
\hline & 8 & 17,1 & 26,3 & 28,4 & 24,2 & 1027 & 245,9 & 256,0 & 579 \\
\hline & 9 & 17,8 & 27,7 & 30,0 & 25,9 & 1020 & 276,6 & 286,7 & 580 \\
\hline & 10 & 18,3 & 29,0 & 31,4 & 27,3 & 1012 & 307,8 & 317,4 & 580 \\
\hline \multirow[t]{9}{*}{1800} & 2 & 9,8 & 12,7 & 13,8 & 8,2 & 1075 & 28,8 & 41,5 & 458 \\
\hline & 3 & 12,0 & 16,4 & 17,7 & 12,4 & 1067 & 62,4 & 73,8 & 458 \\
\hline & 4 & 13,7 & 19,5 & 21,0 & 16,0 & 1059 & 114,0 & 125,2 & 468 \\
\hline & 5 & 15,0 & 22,1 & 23,8 & 19,0 & 1051 & 156,2 & 166,9 & 494 \\
\hline & 6 & 16,1 & 24,3 & 26,3 & 21,7 & 1043 & 200,7 & 211,1 & 522 \\
\hline & 7 & 17,0 & 26,3 & 28,4 & 24,0 & 1035 & 242,8 & 253,0 & 550 \\
\hline & 8 & 17,7 & 28,0 & 30,3 & 26,0 & 1027 & 280,6 & 290,5 & 580 \\
\hline & 9 & 18,4 & 29,5 & 32,0 & 27,7 & 1020 & 317,6 & 327,5 & 579 \\
\hline & 10 & 19,0 & 30,9 & 33,5 & 29,3 & 1012 & 353,8 & 363,5 & 578 \\
\hline \multirow[t]{9}{*}{2300} & 2 & 10,0 & 13,2 & 14,4 & 8,7 & 1075 & 24,7 & 36,0 & 458 \\
\hline & 3 & 12,3 & 17,2 & 18,5 & 13,1 & 1067 & 75,9 & 87,1 & 458 \\
\hline & 4 & 14,0 & 20,4 & 22,0 & 16,8 & 1059 & 127,1 & 137,8 & 470 \\
\hline & 5 & 15,4 & 23,1 & 24,9 & 20,0 & 1051 & 174,5 & 185,0 & 495 \\
\hline & 6 & 16,5 & 25,4 & 27,5 & 22,8 & 1043 & 219,9 & 230,1 & 523 \\
\hline & 7 & 17,4 & 27,5 & 29,7 & 25,2 & 1035 & 267,5 & 277,4 & 550 \\
\hline & 8 & 18,2 & 29,2 & 31,7 & 27,3 & 1027 & 310,8 & 321,0 & 579 \\
\hline & 9 & 18,9 & 30,8 & 33,5 & 29,2 & 1020 & 351,7 & 361,5 & 578 \\
\hline & 10 & 19,5 & 32,2 & 35,1 & 30,8 & 1012 & 385,4 & 395,2 & 575 \\
\hline
\end{tabular}


Tabela 4: Estimativa do carbono e $\mathrm{CO} 2$ seqüestrados na idade de 7 anos em função das condições de crescimento esperadas para cada classe de precipitação pluviométrica anual (t.ha-1) em povoamento clonais de Eucalyptus grandis

Table 4: Estimation of carbon and $\mathrm{CO}_{2}$ stored in the biomass of a 7-year clonal Eucalyptus grandis stand as a function of annual precipitation

\begin{tabular}{|c|c|c|c|c|c|}
\hline \multirow[b]{2}{*}{ Estimativa de } & \multirow[b]{2}{*}{ Compartimento } & \multicolumn{4}{|c|}{ Precipitação anual (mm) } \\
\hline & & 800 & 1300 & 1800 & 2300 \\
\hline \multirow{6}{*}{ Carbono (t/ha) } & Raiz & 7,2 & 9,0 & 10,3 & 11,3 \\
\hline & Lenho & 48,9 & 61,0 & 70,5 & 77,4 \\
\hline & Casca & 4,2 & 5,3 & 6,1 & 6,7 \\
\hline & Copa & 2,3 & 2,9 & 3,4 & 3,7 \\
\hline & Serapilheira & 3,3 & 4,1 & 4,7 & 5,2 \\
\hline & TOTAL & 65,9 & 82,3 & $\mathbf{9 5 , 1}$ & 104,3 \\
\hline \multirow{6}{*}{ CO2 (t/ha) } & Raiz & 26,3 & 32,9 & 38,0 & 41,6 \\
\hline & Lenho & 179,4 & 224,0 & 258,8 & 283,9 \\
\hline & Casca & 15,5 & 19,4 & 22,4 & 24,5 \\
\hline & Copa & 8,6 & 10,7 & 12,4 & 13,6 \\
\hline & Serapilheira & 12,1 & 15,1 & 17,4 & 19,1 \\
\hline & TOTAL & 241,9 & 302,1 & 348,9 & 382,8 \\
\hline
\end{tabular}

Uma consideração importante do ponto de vista de seqüestro de carbono diz respeito ao destino de cada compartimento após o corte das árvores. Toda matéria que seja utilizada para fins energéticos acaba retornando para a atmosfera na forma de $\mathrm{CO}_{2}$. As partes deixadas no local, como raízes, serapilheira, copa e por vezes a casca, ou se mineralizam prendendo-se à matéria orgânica do solo ou retornam com o tempo à atmosfera via decomposição. Considerando que a madeira do tronco é utilizada até $7 \mathrm{~cm}$ com casca na produção de celulose e o restante normalmente é utilizado como combustível em fornos e caldeiras, é importante separar a fração comercial do tronco. A Figura 2 apresenta a curva de crescimento do carbono seqüestrado para a porção comercial e total do tronco, bem como para os demais compartimentos da biomassa do povoamento, na condição de $1.800 \mathrm{~mm}$ de precipitação anual.

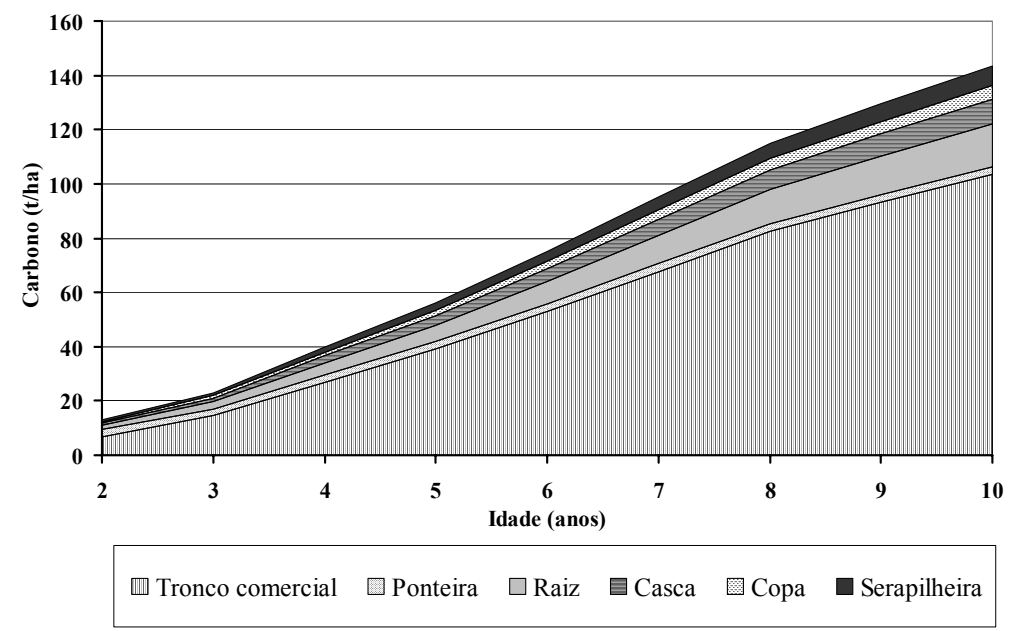

Figura 2: Curva de crescimento de carbono seqüestrado nos diversos compartimentos da floresta, para uma condição de $1.800 \mathrm{~mm}$ de precipitação anual

Figure 2: Growth curve of carbon stored in different compartments of the forest stand under a 1,800 $\mathrm{mm}$ annual precipitation 
Projetos de florestamento e reflorestamento têm uma grande possibilidade de serem implantados em locais ocupados anteriormente por pastagens. Neste caso o cenário de referência (baseline) de seqüestro de carbono poderia ser considerado em estabilidade, ou seja, entradas iguais às saídas, pois todo o pasto em crescimento estaria sendo utilizado como alimento pelo gado ou queimado anualmente. Conseqüentemente o cenário de referência de seqüestro de carbono seria zero. Assim optou-se considerar as produções estimadas advindas do reflorestamento como sendo valores líquidos de carbono seqüestrado (projeto menos cenário de referência), conforme regras para elegibilidade de projetos. Também não se considerou no estudo algumas fontes de liberação de carbono pela operação florestal, como no preparo do solo para plantio (alguma quantidade do carbono armazenado no solo é liberado) e a energia fóssil gasta pelas diversas máquinas de motor à combustão.

\section{Viabilidade de um projeto florestal considerando o seqüestro de carbono}

A tabela 5 apresenta os resultados obtidos de taxa interna de retorno para a parte do lenho comercial e a Tabela 6 para todos os compartimentos da floresta diante de três cenários de preços de tonelada de carbono: 7 , 12 e 17 dólares por tonelada.

Tabela 5: Taxas Internas de Retorno para a parte do lenho comercial

Table 5: Internal Rate of Return for carbon stored in the bole

Lenho Comercial

\begin{tabular}{cccc}
\hline & \multicolumn{3}{c}{ Preço do Carbono (US\$/t) } \\
\cline { 2 - 4 } IMA & $\mathbf{7 , 0 0}$ & $\mathbf{1 2 , 0 0}$ & $\mathbf{1 7 , 0 0}$ \\
\hline $\mathbf{m 3 s c} / \mathbf{h a} . \mathbf{0 n o})$ & $-17,1$ & $-7,2$ & $-0,09$ \\
$\mathbf{2 4 , 0}$ & $-12,8$ & $-3,0$ & 3,3 \\
$\mathbf{3 0 , 0}$ & $-10,1$ & $-0,3$ & 6,0 \\
$\mathbf{3 4 , 7}$ & $-8,3$ & 1,4 & 7,7 \\
$\mathbf{3 8 , 2}$ & & & \\
\hline
\end{tabular}

Tabela 6: Taxas Internas de Retorno para todos os compartimentos

Table 6: Internal Rate of Return for carbon stored in all compartments

Todos

\begin{tabular}{cccc}
\hline & \multicolumn{3}{c}{ Preço do Carbono (US\$/t) } \\
\cline { 2 - 4 } IM3sc/ha.ano) & $\mathbf{7 , 0 0}$ & $\mathbf{1 2 , 0 0}$ & $\mathbf{1 7 , 0 0}$ \\
\hline $\mathbf{2 4 , 0}$ & $-10,5$ & $-0,8$ & 5,5 \\
$\mathbf{3 0 , 0}$ & $-6,5$ & 3,2 & 9,5 \\
$\mathbf{3 4 , 7}$ & $-3,9$ & 5,8 & 12,2 \\
$\mathbf{3 8 , 2}$ & $-2,3$ & 7,5 & 13,9 \\
\hline
\end{tabular}

Como pode se observar nas Tabelas 5 e 6 , valores de US\$ 7,00/tC não permitem atingir valores positivos de taxa interna de retorno, mesmo em situações de alta produtividade e considerando todos os compartimentos de biomassa da floresta. Produtividades abaixo de $30 \mathrm{~m}^{3} \mathrm{sc} \cdot \mathrm{ha}^{-1}$.ano ${ }^{-1}$ são pouco atrativas economicamente se a intenção é somente seqüestrar carbono. Nesse nível de produção, a taxa interna de retorno para o lenho comercial alcançaria $3,3 \%$ ao ano quando o preço da tonelada de carbono seqüestrado fosse de US\$ 17,00/tC. Considerando todos os compartimentos da floresta a taxa interna de retorno seria de US\$ 9,50/tC.

Produtividades superiores a 34,7 $\mathrm{m}^{3} \mathrm{sc} /$ ha.ano são mais atrativas, porém, somente preços de US\$17,00/tC promovem atratividades do lenho comercial que ultrapassem as aplicações financeiras comuns de mercado, como a remuneração da caderneta 
de poupança ( $6 \%$ ao ano). No caso de todos os compartimentos da floresta, o preço mínimo que garante uma rentabilidade acima desse nível é de US\$ 12,00/tC para produtividades superiores a $34,7 \mathrm{~m}^{3} \mathrm{sc} \cdot \mathrm{ha}^{-1}$. ano $^{-1}$. Valores de US\$ 17,00 remuneram de forma economicamente atrativa até quando as produtividades são menores $\left(24 \mathrm{~m}^{3} \mathrm{sc} \cdot \mathrm{ha}^{-1} \cdot \mathrm{ano}^{-1}\right)$.

Dependendo das regras a serem definidas para o mercado de carbono, a possibilidade de comercialização da madeira para usos sólidos que não impliquem na liberação do carbono seqüestrado para a atmosfera poderá complementar a rentabilidade do empreendimento. Adotando valor da madeira em pé (lenho comercial) de US $\$ 8,00 / \mathrm{m}^{3} \mathrm{sc}$ e de carbono a US $\$ 12,00 / \mathrm{tC}$, a rentabilidade é aumentada para todas as classes de produtividade, conforme apresenta a tabela 7 .

Tabela 7: Atratividade econômica dada pela Taxa Interna de Retorno considerando valor da madeira e de carbono (em \%)

Table 7: Economical atractivity given by the Internal Rate of Return taking into account wood and carbon values

\begin{tabular}{llccc}
\hline & \multicolumn{4}{c}{ Produtividade IMA7 $\mathbf{~ m}^{\mathbf{3}} \mathbf{s c} / \mathbf{h a . a n o}$} \\
Compartimento & $\mathbf{2 4 , 0}$ & $\mathbf{3 0 , 0}$ & $\mathbf{3 4 , 7}$ & $\mathbf{3 8 , 2}$ \\
\hline Lenho comercial & 15,2 & 19,5 & 22,3 & 24,2 \\
Todos os compartimentos & 17,4 & 21,7 & 24,5 & 26,4 \\
\hline
\end{tabular}

Considerando a possibilidade de venda da porção lenhosa, sem prejuízo das bases contratadas pelo seqüestro de carbono, mesmo produtividades baixas gerariam taxas internas de retorno acima de diversas opções do mercado financeiro, com valores superiores a $15 \%$ ao ano. A inclusão dos demais compartimentos da floresta na negociação envolvendo seqüestro de carbono acrescenta pouca atratividade relativa e, face às inerentes dificuldades de monitoramento e validação das estimativas dos compartimentos (raiz, folha, galho e serapilheira), poderá ter sua inclusão questionada.

Uma outra questão importante para melhor entendimento sobre a valoração justa do carbono seqüestrado por reflorestamento é apresentada na Tabela 8. Optou-se por simular o preço de contribuição de cada compartimento da biomassa de forma a obtenção de remuneração equivalente ao da caderneta de poupança ( $6 \%$ ao ano), para cada classe de produtividade.

Tabela 8: Valor estimado do carbono seqüestrado pelos diversos compartimentos da biomassa (US\$/tC) por classe de produtividade e para rentabilidade de $6 \%$ ao ano

Table 8: Value of carbon stored in different biomass compartments (US\$ $/ \mathrm{tC}$ ) by yield class and for a feasibility rate of $6 \%$ a year

\begin{tabular}{cccccc}
\hline \multirow{3}{*}{ Estimativa de } & Compartimento & $\mathbf{2 4 , 0}$ & $\mathbf{3 0 , 0}$ & $\mathbf{3 4 , 7}$ & $\mathbf{3 8 , 2}$ \\
\hline & LC & 25,0 & 19,8 & 17,0 & 15,5 \\
& LC+LP & 23,5 & 19,0 & 16,4 & 14,9 \\
Carbono (t/ha) & LC+LP+CA & 21,7 & 17,4 & 15,1 & 13,7 \\
& LC+LP+CA+CO & 20,8 & 16,7 & 14,4 & 13,1 \\
& LC+LP+CA+CO+RA & 18,4 & 14,8 & 12,8 & 11,7 \\
& LC+LP+CA+CO+RA+SE & 17,5 & 14,0 & 12,1 & 11,1 \\
\hline
\end{tabular}

LC - lenho comercial; LP - lenho da ponteira; CA - casca; CO - copa; RA - raiz; SE - serapilheira 
O preço da tonelada de carbono seqüestrado que remunera o investimento a $6 \%$ ao ano varia de US\$ $11,10 / \mathrm{tC}$ até US\$ 25,00/t/C, para diferentes produtividades e compartimentos da biomassa. Quando somente se considera o lenho comercial para efeito do seqüestro de carbono, as produtividades menores implicam em valores acima da realidade atualmente esperada para este mercado. Já quando se consideram todos os compartimentos da floresta, a rentabilidade de $6 \%$ ao ano pode ser garantida aos preços vigentes, independentemente da produtividade do local.

O empreendimento florestal que objetive seqüestro de carbono exclusivamente necessitará incluir todos os compartimentos da biomassa na pauta de negociação para que se obtenha uma rentabilidade econômica semelhante ao da caderneta de poupança, a preços dentro da faixa esperada (até US\$ $20,00 / \mathrm{tC}$ ). A produtividade do povoamento como função da situação climática local influi nos preços do carbono seqüestrado. Produtividades acima de $30 \mathrm{~m}^{3} \mathrm{sc} \cdot \mathrm{ha}^{-1}$.ano-1 já permitem rentabilidade de $6 \%$ ao ano dentro dos preços atualmente esperados (até US\$ 20,00/tC), mesmo se for considerado somente a porção comercial do lenho das árvores.

\section{CONCLUSÕES}

- A condição climática, traduzida pela média histórica da precipitação anual, interfere na quantidade de carbono a ser seqüestrado. Para locais com $800 \mathrm{~mm}$ de precipitação anual estima-se um total de $65,9 \mathrm{tC} / \mathrm{ha}$ em todos os compartimentos da floresta (raiz, tronco, copa, casca e serapilheira) aos 7 anos de idade. Já para locais com 2.300 $\mathrm{mm}$ anuais, essa quantidade sobe para $104,3 \mathrm{tC} / \mathrm{ha}$, correspondendo a um aumento de $58 \%$;

- Na faixa de preços avaliada (entre US\$ 7,00 e US\$ $17,00 / \mathrm{tC}$ ), que é esperada atualmente no mercado de carbono, não são vislumbradas rentabilidades acima de $7,7 \%$ ao ano considerando seqüestro somente da porção do lenho comercial (tronco até $7 \mathrm{~cm}$ com casca). As rentabilidades são melhores somente com preços de carbono de US\$ 17,00/tC e produtividades florestais acima de 34,7 $\mathrm{m}^{3} \mathrm{sc} \mathrm{ha}^{-1} \cdot$ ano $^{-1}$ aos 7 anos de idade. Se o seqüestro de carbono for considerado em todos os compartimentos da biomassa, então preços médios de US\$12,00/tC e produtividades acima de $30 \mathrm{~m}^{3} \mathrm{sc} \cdot \mathrm{ha}^{-1} \cdot \mathrm{ano}^{-1}$ resultam em taxas internas de retorno superiores a $6 \%$ ao ano;

- Caso a madeira resultante possa ser comercializada sem prejuízo das bases negociadas para seqüestro de carbono, as rentabilidades tornar-se-iam mais atrativas. $\mathrm{Se}$ somente o lenho comercial fosse vinculado ao seqüestro de carbono, então as taxas internas de retorno variariam de $15,2 \%$ ao ano em locais com precipitação anual histórica de $800 \mathrm{~mm}$, até $24,2 \%$ ao ano em locais com $2.300 \mathrm{~mm}$ anuais. Se todos os compartimentos da biomassa fossem levados em conta para fins de seqüestro de carbono, então os valores de TIR variariam de $17,4 \%$ a $26,4 \%$ ao ano, respectivamente;

- O preço do carbono seqüestrado que garante uma atratividade mínima de $6 \%$ ao ano varia de acordo com a produtividade florestal e de quais compartimentos da biomassa serão levados em conta no projeto. Quando somente o lenho comercial for considerado para seqüestro de carbono, os preços a serem praticados deveriam ser de US\$25,00/tC, para locais com incremento médio anual em volume de madeira aos 7 anos (IMA7) de 24 $\mathrm{m}^{3}$ sc.ha ${ }^{-1}$.ano ${ }^{-1}$ e corte aos 7 anos, até US\$ 15,50/tC, para locais com IMA7 de 38,2 $\mathrm{m}^{3} \mathrm{sc} \mathrm{ha}^{-1}$. ano ${ }^{-1}$. Preços mais próximos aos esperados (US\$ 12,00/tC) pelo mercado somente são possíveis se toda a biomassa for considerada no projeto de carbono e produtividades acima de $34,7 \mathrm{~m}^{3} \mathrm{sc}$.ha ${ }^{1}$.ano 1 com corte aos 7 anos forem atingidas;

- A união de ferramentas quantitativas para projeção das características do povoamento florestal possibilita a estimativa da produção florestal e do carbono possível de ser seqüestrado por 
povoamentos clonais de Eucalyptus grandis. No mercado futuro de carbono, onde expectativas de seqüestro em áreas atualmente sem plantios serão demandadas, tais ferramentas tornar-se-ão operacionalmente úteis.

\section{REFERÊNCIAS}

Cannell, M.G.R. Growing trees to sequester carbon in UK: answers to some common questions. Institute of Terrestrial Ecology, Bush State, Penicuik, Scotland. Forestry, v. 72, n.3, p. 237-247, 1999.

Goldenberg, J. O Brasil e as mudanças climáticas. O Estado de São Paulo, 12/12/1999.

Gutiérrez, V.H.; Lopera, G.J. Flujo de carbono y respuesta a diferentes estratégias de manejo en plantaciones tropicales de Pinus patula. In: Simpósio Internacional: Medición y Monitoreo de Carbono em Ecositemas Forestales, Valdivia-Chile, 2001.

Maestri, R. Modelo de crescimento e produção implícito para povoamentos de híbridos naturais de Eucalyptus grandis baseado em variáveis do ambiente e do povoamento. Curitiba: Programa de PósGraduação em Engenharia Florestal (Doutorado em Ciências Florestais), Universidade Federal do Paraná, 2003 (no prelo).

Neves, J.C.L. Produção e partição de biomassa, aspectos nutricionais e hídricos em plantios clonais de eucalipto na região litorânea do Espírito Santo. Campos dos Goytacazes: Programa de Pós-Graduação em Ciências e Tecnologias Agropecuárias (Doutorado em Produção Vegetal), Universidade Estadual do Norte Fluminense, 2000. $191 \mathrm{p}$.
Nutto, L.; Watzlawick, L.F.; Grammel, R.; Fenner, P.T. O mercado internacional de $\mathrm{CO}_{2}$ : Impacto das florestas naturais e plantações. In: As Florestas e o Carbono. Curitiba: UFPR, 2002. p. $89-108$.

Ribeiro, L.S.; Oliveira, A S.; Novaes, E.S.; La Rovere, E. L.; Pereira, A S.; Dubeux, C.B.S.; Costa, C.V.; Oliveira, L.B; Muylaert, M.S. Proposta de critérios e indicadores de elegibilidade para avaliação de projetos candidatos ao mecanismo de desenvolvimento limpo (MDL). In: As Florestas e o Carbono. Curitiba: UFPR, 2002. p. 35-58.

Rocha, M.T. O aquecimento global e os instrumentos de mercado para a solução do problema. In: As Florestas e o Carbono. Curitiba: UFPR, 2002. p.1 - 34.

Sanquetta, C.R. Métodos de determinação de biomassa florestal. In: As Florestas e o Carbono. Curitiba: UFPR, 2002. p. 119-140.

Scolforo, J.R.S. Biometria Florestal Modelagem do crescimento e da produção de florestas plantadas e nativas. Lavras: UFLA/FAEPE/DCF, 441 p. Ano 1998.

Stape, J.L. Production ecology of clonal eucalyptus plantation on northeastern Brazil. Fort Collins, Colorado - EUA: Programa de Pós-Graduação em Ciência Florestal (Ph.D. Thesis in Forest Ecology), Colorado State University, 2002. 225 p.

Thiersch, C.R. Modelagem da densidade básica, do volume e do peso seco para plantios de clones de Eucalyptus sp. Lavras: Programa de Pós-Graduação em Engenharia Florestal (Mestrado em Engenharia Florestal), Universidade Federal de Lavras, 2002 197p. 